\title{
Limites de la notion de dose collective $(*)$
}

\author{
S. VIGNES, M. BERTIN, G. WOLBER $(* *)$ \\ (Manuscrit reçu le 24 août 1981)
}

\begin{abstract}
RÉSUMÉ
La dose collective, notion apparue en radioprotection vers la fin des années 1960, a rendu de grands services au plan opérationnel, permettant à la fois de faire des bilans de doses ponctuels ou globaux et de faire des choix technologiques en vue d'économiser les doses.

L'utilisation de la dose collective apparaît très discutable pour additionner des doses de groupes irradiés à des niveaux très différents et surtout pour calculer les dommages correspondants et faire des intercomparaisons entre différents groupes. La remise en cause de la relation linéaire dans le domaine des très faibles doses ( $\leqq 10$ rems) et des faibles débits de dose, qui est aussi celui qui intéresse les doses collectives des travailleurs ou de la population, amène à minimiser d'autant plus le dommage que la dose est plus faible. Alors, les doses collectives de la population deviennent totalement négligeables par rapport aux doses collectives des travailleurs, sur lesquelles doivent porter tous les efforts de radioprotection.
\end{abstract}

\begin{abstract}
The concept of the collective dose has been useful at the operational level, for both punctual or global dose assessments and technological choices in order to avoid exposures.

In order to add up the doses to groups exposed at very different levels and still more to evaluate the corresponding damage and make intercomparisons between various groups, the use of the collective is open to discussion. Questioning the linear relationship at very low doses ( $\leqq 10$ rems) and low dose rates - which also concern the collective doses to workers or to the population-could minimize the damage as the dose is lower. The collective doses to the population then become insignificant as compared to the collective doses to workers with which radiation protection measures should be most concerned.
\end{abstract}

(*) Communication présentée lors du Séminaire sur les progrès dans les techniques de radioprotection des travailleurs de l'énergie nucléaire, Cadarache, 5-7 mai 1981.

(**) Électricité de France, Comité de Radioprotection, 39, rue de Washington, 75384 Paris Cedex 08.

RADIOPROTECTION, VOL. $16-0033-8451 / 1981 / 255 / \$ 5.00 /$ C Bordas-Dunod. 


\section{CARACTÉRISTIQUES ET DÉFINITION DE LA DOSE COLLECTIVE}

La notion de dose collective est utilisée en radioprotection depuis la fin des années 1960. Une dose collective est la simple somme des doses individuelles. individuelles.

Donc : $D_{c}=d_{1}+d_{2}+d_{3}+\ldots+d_{12}=\sum_{i=1}^{n} d_{i}$, où $d_{i}$ représente les doses

Elle a longtemps été exprimée en homme-rems et suivant la définition donnée par Sinderman et Axtelle en 1969 [1] : " un homme-rem est toute combinaison de doses individuelles dont le total est de $1 \mathrm{rem}$; par exemple, un homme recevant $1 \mathrm{rem}$, ou quatre hommes recevant un quart de rem, ou mille recevant 1 mrem, etc. ". Depuis la définition d'une nouvelle unité d'équivalent de dose, le sievert, centuple du rem, l'expression de la dose collective est l'homme-sievert. Un homme sievert est donc égal à 100 homme-rems. Nous nous exprimerons ici encore en homme-rems. La dose individuelle moyenne est obtenue évidemment en divisant la dose collective par l'effectif du groupe considéré. La justification de cette notion est donnée par les recommandations de la Commission Internationale de Protection Radiologique (C.I.P.R.) [2, 3].

Cependant, il nous semble qu'on englobe sous ce terme deux notions un peu différentes. Dans certains cas, la dose collective est utilisée sans qu'on la traduise en dommage ou en détriment; additionner les doses individuelles, comparer les doses collectives de groupes différents ne pose pas de problème. Dans d'autres cas, la notion de dose collective est prise, de façon implicite, dans le sens d'un " équivalent de dose " collectif et permet de calculer des dommages. Ceci n'a de sens que si l'on admet, comme seule relation dose-effet possible, la relation linéaire sans seuil. En effet, cela suppose à tout niveau de dose, une stricte proportionnalité entre l'effet et la dose (si un effet $E$ correspond à une dose $D$, il faut qu'en multipliant ou en divisant la dose par un facteur $k$, l'effet soit multiplié ou divisé par le même facteur $k$ ). Sinon, l'erreur serait variable suivant le niveau de dose et, en toute rigueur, additionner les doses et les traduire en dommage collectif n'aurait pas grand sens. Il faut aussi admettre que cette relation doseeffet n'est pas modifiée par les conditions d'irradiation (débit de dose, fractionnement, etc.), les caractéristiques des sujets exposés, etc.

C'est, en effet, à partir de ces deux hypothèses qu'on calcule le dommage; la valeur en est la même pour la dose collective que pour la somme des doses le composant. En fait, la méthode de calcul global à partir de la dose collective apparaît plus aisée et plus rapide que celle qui consisterait à additionner les dommages estimés individuellement.

La C.I.P.R. recommande donc l'usage des doses collectives pour l'estimation des dommages collectifs correspondants, permettant de remonter ensuite au détriment, c'est-à-dire au dommage pondéré par sa gravité.

\section{QUEL EST LE DOMAINE DE DOSES COUVERT PAR LA DOSE COLLECTIVE ?}

Dans la majorité des cas, qu'il s'agisse de travailleurs ou de la population générale, le domaine des doses individuelles intéressé par le calcul de la dose 
collective est celui des faibles doses, depuis des fractions de millirem jusqu'à 10 rems, soit des variations de 1 à plus de 10000 . Il s'agit, dans tous les cas, de doses prises annuellement, donc, en réalité, plutôt d'un débit de dose annuel. C'est là le domaine où existe le maximum d'incertitudes sur le type convenable de relation entre la dose et l'effet. On conçoit, par conséquent, que l'on doive examiner avec prudence le résultat de l'appréciation du dommage en résultant.

\section{UTILITÉ DE LA DOSE COLLECTIVE}

La notion de dose collective est évidemment très utile en radioprotection car elle permet des comparaisons intéressantes. Celles-ci portent sur les doses et non sur les dommages. On peut presque dire qu'il s'agit de la comparaison des doses absorbées collectives. Sur un site nucléaire, elle permet de faire des bilans dosimétriques.

Ces bilans peuvent être ponctuels, concernant une intervention donnée, par exemple les opérations répétitives chaque année, au moment de la révision d'une tranche et du rechargement. Cela permet de suivre les progrès effectués pour essayer de réduire les doses, par exemple grâce à une meilleure préparation des interventions ou grâce à des aides technologiques diverses. Les doses collectives inhérentes à chaque opération peuvent être rapportées au temps nécessité par l'opération et pour certaines interventions, en général de courte durée, leur coût dosimétrique permet d'aider à estimer le nombre d'opérateurs optimal.

Les bilans concernent aussi l'ensemble des travaux d'un site et sont alors annuels; ils permettent des intercomparaisons entre tranches ou sites nucléaires ou des études de l'évolution pour chaque site au cours du temps. Ces comparaisons sont valables s'il s'agit d'opérations similaires et si les domaines de doses sont les mêmes.

Enfin, les experts de l'U.N.S.C.E.A.R. [4] se sont servis de cette notion pour faire des bilans de doses reçues par le public et les travailleurs, rapportés au mégawatt électrique produit. Il s'agit alors d'un bilan global, tenant compte de l'ensemble du cycle du combustible.

L'intérêt de la dose collective est donc d'être un guide pour l'action et pour la prévention. La comparaison entre deux méthodes distinctes d'intervention qui se sont soldées par deux valeurs distinctes de dose collective permet de retenir celle qui aboutit à la plus grande économie de doses.

Ceci, lorsqu'on approfondit les choses, doit être nuancé et le choix qui sera fait devra tenir compte à la fois des doses individuelles et des doses collectives. Un exemple permettra de la comprendre.

Une intervention dans une boîte à eau de générateur de vapeur, dans laquelle le débit de dose est de 10 à $20 \mathrm{rem} / \mathrm{h}$, induit des doses au moment de la pénétration dans la boîte à eau, durant l'intervention proprement dite et lors de la sortie de la boîte à eau.

La dose collective peut être exprimée de la façon suivante :

$D=$ dose nécessitée par l'intervention $+N$ (dose entrée + dose sortie), où $N$ est le nombre d'agents intervenant. 
Toute réduction de la dose individuelle revient à augmenter $N$, donc la dose collective. Cette augmentation peut être importante si les doses entrée et sortie sont elles-mêmes importantes.

De plus, si l'on hésite à modifier une procédure, par exemple pour le traitement des effluents, le coût en dose collective peut être mis en balance avec le coût économique de la nouvelle technique.

Mais ici s'arrête, à notre avis, l'intérêt de cette notion car tout calcul d'optimisation exigerait que l'on puisse traduire avec exactitude la dose collective en dommages ou en détriment.

\section{CRITIQUE DE L'UTILISATION DE LA DOSE COLLECTIVE}

La traduction en dommages de la dose collective doit être très prudente. Elle est même discutable pour faire des intercomparaisons entre doses collectives de populations ayant reçu des niveaux de doses très différents. De telles intercomparaisons, pour être valables, supposeraient que l'on apprécie de façon exacte les dommages dans les deux cas et que l'on connaisse avec certitude la relation dose-effet correspondant à ce domaine des doses.

Plusieurs critiques peuvent d'abord être faites à propos des caractéristiques mêmes de la dose collective, et elles mettent en question le calcul d'un dommage correspondant et les comparaisons de ces dommages, dans les différents cas de figure.

\section{1. HÉtÉROGÉNÉITÉ DES DOSES A L'INTÉRIEUR DES GROUPES OU ENTRE GROUPES}

Si l'on calcule, par exemple, une dose collective pour les travailleurs durant 1 année, on sera amené à additionner des doses de l'ordre de quelques dizaines de millirems avec des doses qui peuvent dépasser 1 rem et atteindre, à la limite, 12 rems. De même, pour la population tout entière, on sera amené à ajouter des millirems (de la population « critique ») à des fractions de millirem, de l'ordre du centième de millirem pour la majeure partie de la population.

Ainsi, à l'intérieur même du groupe étudié, existe déjà une grande hétérogénéité de doses (1 à 100) mais il est évident que cela est encore beaucoup plus criant lorsqu'on compare ou lorsqu'on ajoute des doses travailleurs à des doses population : la variation est alors de 1 à plus de 10000 .

\section{2. DifFÉRENCES INHÉRENTES AU MODE D’OBTENTION DES DONNÉES DOSIMÉTRIQUES}

Lorsqu'on calcule une dose collective, deux cas peuvent se présenter : ou bien, comme c'est le cas pour les travailleurs du nucléaire, l'on a déterminé les doses par des mesures effectives à l'aide d'une dosimétrie individuelle (par films ou par d'autres moyens), ou bien, comme pour la population, l'on a évalué les doses par calcul, selon des modèles de transfert appropriés au cas étudié. Les doses ainsi estimées dépendent étroitement du choix de ces modèles et l'on 
conçoit qu'il existe une imprécision dans cette évaluation. Les imprécisions inhérentes au calcul même de la dose collective rendent fragile l'utilisation de ce critère à des fins de calcul sanitaire.

\subsection{Hétérogénéité de la nature de Rayonnements}

Les rayonnements auxquels les groupes peuvent être exposés sont divers : irradiation externe par photons gamma ou $\mathrm{X}$, neutrons ou irradiation interne par émissions bêta ou alpha.

En fait, la majorité des expositions concerne des rayonnements à faible densité d'énergie. Ce sont les émetteurs gamma (cobalt 60, césium 134 et 137, etc.) et bêta (strontium 90, ruthénium 106) qui jouent le rôle prépondérant pour la dose communiquée au public. Dans le cas d'une irradiation professionnelle, il s'agit surtout encore de bêta et de gamma.

Les émissions $\alpha$ sont beaucoup plus rares : citons le cas du radon, qui constitue le risque auquel sont soumis les mineurs d'uranium. Les neutrons ne concernent que le " bâtiment réacteur " des centrales en fonctionnement.

Cependant, la dose collective est due à des rayonnements divers, auxquels on ne peut attribuer les mêmes valeurs de dommages.

\subsection{Choix de la Relation dose-efFet Convenable}

Rappelons que le domaine de doses couvert par les calculs de dose collective est celui des faibles doses, dans tous les cas inférieures à 10 rems. Comme nous l'avons déjà souligné, il s'agit d'un débit de dose annuel, aussi bien pour les travailleurs que pour le public. Dans ce domaine de faibles doses et de faibles débits de dose, selon la majorité des experts, deux faits méritent d'être soulignés :

$1^{\circ}$ La relation linéaire entre la dose et l'effet surestime les risques dans le cas le plus fréquent des rayonnements à bas TLE. Cette surestimation peut, selon les cas, varier d'un facteur 2 à 10 [4, 5]. Le rapport BEIR III [6] a notamment officialisé une relation linéaire-quadratique pour représenter le dommage dans ce cas; celle-ci conduit à réduire d'un facteur 2 environ les évaluations faites par le BEIR de 1972 [7]. Enfın, certains auteurs, comme Rossi, préconisent, aux faibles doses et pour ce type de rayonnements, la relation quadratique.

Cette relation linéaire semble convenir, par contre, dans le cas des rayonnements à TLE élevé. De même, dans ce cas, l'effet du débit de dose est beaucoup moins certain.

$2^{\circ}$ FABRIKANT et Rossi relèvent dans le BEIR III qu'il est, de toute façon, imprudent de se livrer à des calculs de dommages aux doses inférieures à 10 rems, par suite de l'importance des incertitudes à ce niveau de doses.

Malgré ces réserves,nous avons néanmoins rassemblé sur les tableaux I et II quelques estimations de dommages, exprimés par million de personnes et sur la durée de vie, pour des expositions à des doses différentes, soit uniques, soit répétitives, sur toute la vie (70 ans) pour la population, ou sur la vie professionnelle (40 ans) pour les travailleurs et selon le type de modèle adopté. 
TABLEAU 1

NOMBRE DE CANCERS MORTELS CALCULÉS PAR MILLION DE PERSONNES SELON LE MODÉLE ET SELON LA DOSE (ET LE DÉBIT DE DOSE)

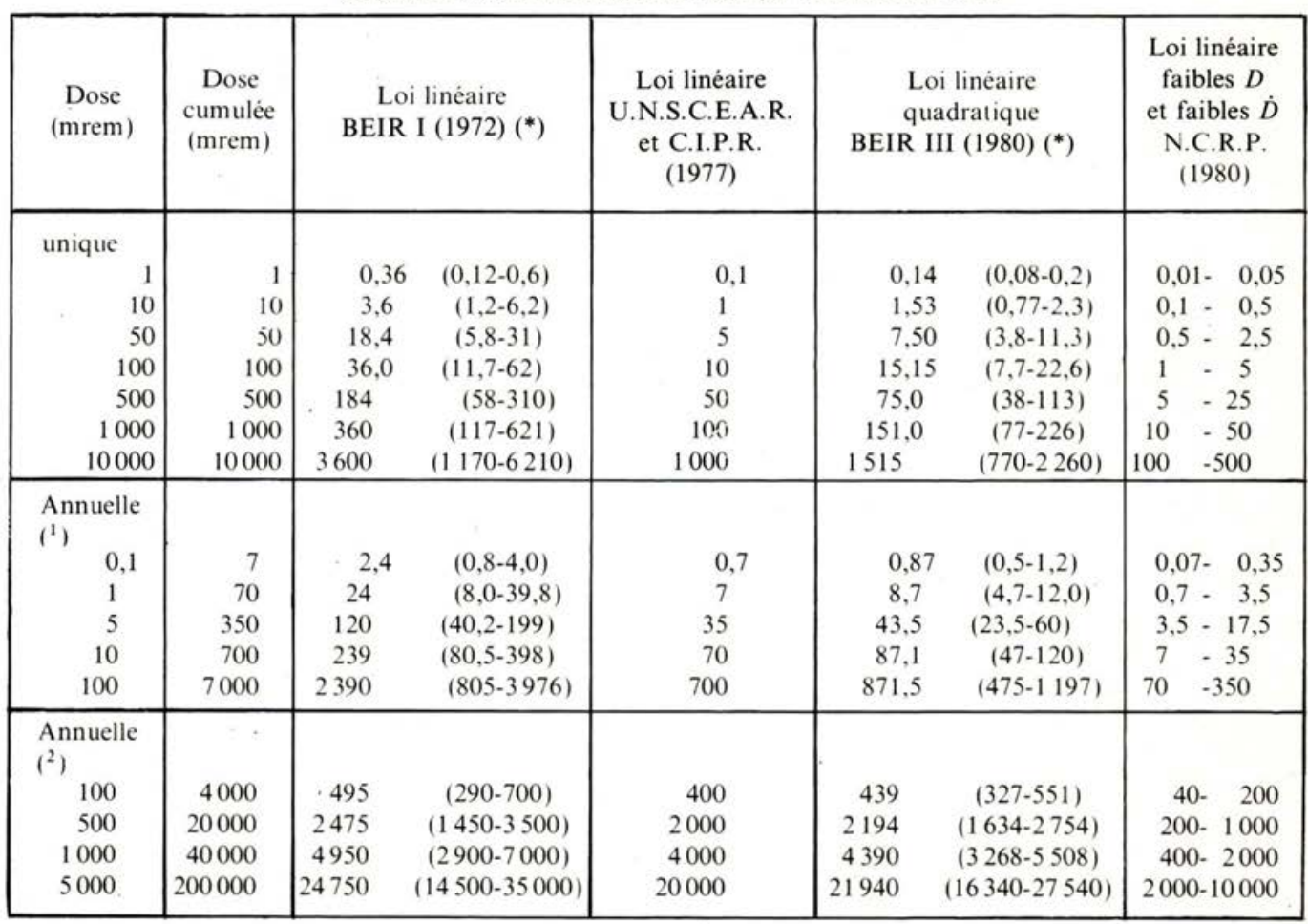

(*) Conformément aux coefficients de risque donnés au tableau V . 25 page 265 du BEIR III [6].

(1) Cas de la population (durée de vie moyenne: 70 ans).

( $\left.{ }^{2}\right)$ Cas des travailleurs (durée d'exposition moyenne : 40 ans).

Le tableau I présente uniquement les modèles linéaires, pondérés ou non par des coefficients tenant compte des faibles niveaux de doses et de débits de dose alors que le tableau II indique, de plus, la relation quadratique. Dans ce dernier cas, les évaluations ont été faites en tenant compte de l'irradiation naturelle de base, estimée à $100 \mathrm{mrem}$, ce qui fait partir la courbe au-delà de l'origine. Ainsi, pour les doses surajoutées relativement très faibles, les variations correspondantes de l'effet sont pratiquement proportionnelles et ne s'écartent de la proportionnalité qu'à partir de 50 à $100 \mathrm{mrem}$ surajoutés, ainsi qu'on peut le voir sur la figure, présentée en échelles log-log.

Selon le modèle retenu, on constate que la valeur du dommage correspondant à une valeur donnée de dose collective peut varier beaucoup : d'un facteur 2 à 10 si l'on adopte exclusivement les modèles linéaires pondérés et d'un facteur d'environ 500 dans le cas de la relation quadratique. 
TABLEAU II

COMPARAISON DES DOMMAGES CALCULÉS POUR UN MILLION DE PERSONNES

SELON LE MODÉLE LINÉAIRE OU QUADRATIQUE

\begin{tabular}{|c|c|c|c|c|}
\hline $\begin{array}{c}\text { Dose } \\
\text { (mrem) }\end{array}$ & $\begin{array}{l}\text { Dose cumulée } \\
\text { (mrem) }\end{array}$ & $\begin{array}{l}\text { Loi linéaire } \\
\text { U.N.S.C.E.A.R. } \\
\text { et C.I.P.R. } \\
(1977)\end{array}$ & $\begin{array}{c}\text { Loi linéaire } \\
\text { faibles } D \\
\text { et faibles } \dot{D} \\
\text { N.C.R.P. }(1980)\end{array}$ & $\begin{array}{c}\text { Loi quadratique Rossi } \\
\text { (100 mrem irradiation } \\
\text { naturelle) }\end{array}$ \\
\hline $\begin{array}{r}\text { Unique } \\
1 \\
10 \\
50 \\
100 \\
500 \\
1000 \\
10000\end{array}$ & $\begin{array}{r}1 \\
10 \\
50 \\
100 \\
500 \\
1000 \\
10000\end{array}$ & $\begin{array}{r}0,1 \\
1 \\
5 \\
10 \\
50 \\
100 \\
1000\end{array}$ & $\begin{array}{c}0,01-0,05 \\
0,1-0,5 \\
0,5-2,5 \\
1-5 \\
5-25 \\
10-50 \\
100-500\end{array}$ & $\begin{array}{c}2-5,6 \cdot 10^{-4} \\
2-6 \cdot 10^{-3} \\
1,2-3,5 \cdot 10^{-2} \\
3-8,4 \cdot 10^{-2} \\
0,35-0,98 \\
1,2-3,4 \\
102-285\end{array}$ \\
\hline $\begin{array}{r}\text { Annuelle }\left({ }^{1}\right) \\
0,01 \\
0,1 \\
1 \\
5 \\
10 \\
100 \\
\end{array}$ & $\begin{array}{r}0.7 \\
7 \\
70 \\
350 \\
700 \\
7000\end{array}$ & $\begin{array}{c}0,07 \\
0,7 \\
7 \\
35 \\
70 \\
700\end{array}$ & $\begin{array}{c}0,007-0,035 \\
0,07-0,35 \\
0,7-3,5 \\
3.5-17.5 \\
7-35 \\
70-350\end{array}$ & $\begin{array}{l}1,4-3,9 \cdot 10^{-4} \\
1,4-3,9 \cdot 10^{-3} \\
1,4-3,9 \cdot 10^{-2} \\
7,2-20 \cdot 10^{-2} \\
0,15-0,41 \\
2.10-5.9\end{array}$ \\
\hline $\begin{array}{c}\text { Annuelle }\left({ }^{2}\right) \\
100 \\
500 \\
1000 \\
5000\end{array}$ & $\begin{array}{r}4000 \\
20000 \\
40000 \\
200000\end{array}$ & $\begin{array}{r}400 \\
2000 \\
4000 \\
20000\end{array}$ & $\begin{array}{c}40-200 \\
200-1000 \\
400-2000 \\
2000-10000\end{array}$ & $\begin{array}{c}1,2-3,4 \\
14,0-39,0 \\
48,0-134 \\
1040-2912\end{array}$ \\
\hline
\end{tabular}

( ${ }^{1}$ ) Cas de la population (durée de vie moyenne: 70 ans).

$\left({ }^{2}\right)$ Cas des travailleurs (durée d'exposition moyenne : 40 ans).

Les inexactitudes inhérentes au mode de calcul du dommage sont d'autant plus importantes que la dose ou plutôt le débit de dose est plus faible.

Donc, s'il est déjà hasardeux de se livrer à un calcul précis de dommage à l'intérieur d'un groupe dont les doses individuelles sont relativement homogènes, a fortiori apparaît-il illogique de vouloir comparer deux doses collectives correspondant à des groupes dont les doses individuelles sont très différentes. Notamment, mettre en balance une dose collective des travailleurs avec une dose collective de la population n'a pas de signification car les incertitudes pour calculer les dommages, dans le second cas, sont beaucoup plus importantes que dans le premier. Par exemple, si l'on examine l'opportunité de mettre en œuvre une nouvelle procédure de traitement des effluents, capable de réduire les rejets à l'environnement tout en provoquant une irradiation supplémentaire des travailleurs, on se trouve tenté de comparer les deux doses collectives correspondantes. Or, la situation ne nous paraît pas du tout comparable : même une ou deux centaines seulement de millirems ajoutés à la 
dose annuelle de quelques travailleurs pèsent beaucoup plus, au plan sanitaire, qu'un millirem (ou fraction de millirem) retranché à la dose d'une population même importante pour des raisons développées plus loin.

\section{NIVEAU D'IRRADIATION DE LA POPULATION \\ IMPUTABLE AU FONCTIONNEMENT DES CENTRALES NUCLÉAIRES}

Les campagnes 1979 et 1980 des récentes tranches PWR en service en France montrent que les rejets représentent souvent une très faible proportion de ce qui était autorisé. Le calcul de la dose à l'organisme entier pour un individu du

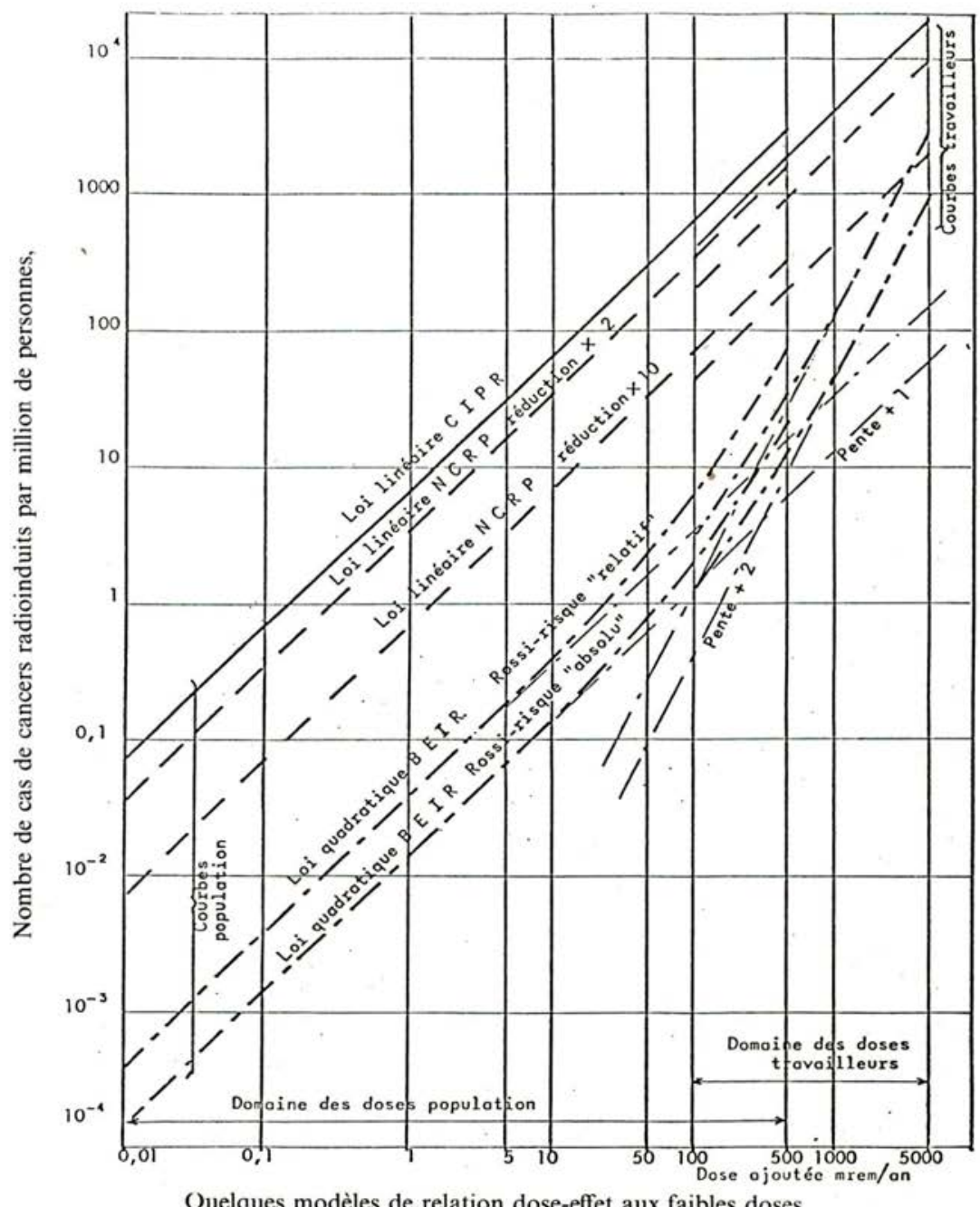

Quelques modèles de relation dose-effet aux faibles doses. 


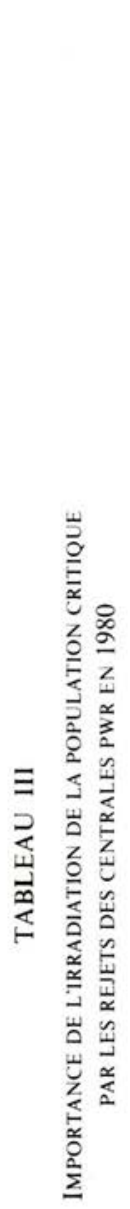

\begin{tabular}{|c|c|c|c|c|}
\hline \multicolumn{3}{|c|}{ 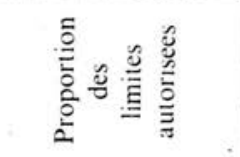 } & $\begin{array}{l}i \\
\stackrel{1}{0} \\
\stackrel{2}{u}\end{array}$ & $\begin{array}{l}\vec{i} \\
\ddot{2} \\
\ddot{0}\end{array}$ \\
\hline \multicolumn{3}{|c|}{ 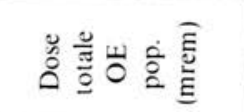 } & $\frac{n}{2}$ & $\frac{0}{0}$ \\
\hline \multirow{2}{*}{ 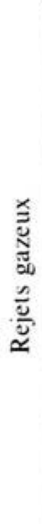 } & \multirow{2}{*}{ 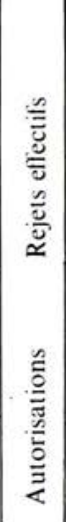 } & 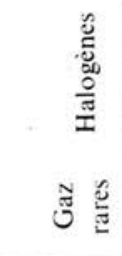 & $\begin{array}{l}\dot{0} \\
\dot{1}\end{array}$ & $\begin{array}{l}\hat{0} \\
\dot{1}\end{array}$ \\
\hline & & 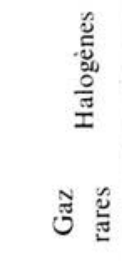 & 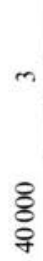 & §్రి \\
\hline \multirow{2}{*}{ 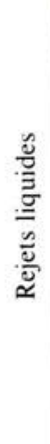 } & \multirow{2}{*}{ 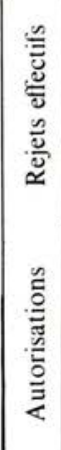 } & 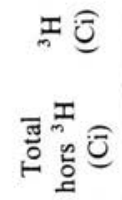 & $\approx$ & $\cong$ \\
\hline & & 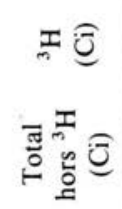 & $\underset{\sim}{8}$ & $\stackrel{8}{8}$ \\
\hline \multicolumn{3}{|c|}{ 总 प } & $N$ & $\overline{\bar{N}}$ \\
\hline \multicolumn{3}{|c|}{$\underset{5}{\check{2}}$} & 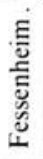 & 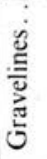 \\
\hline
\end{tabular}

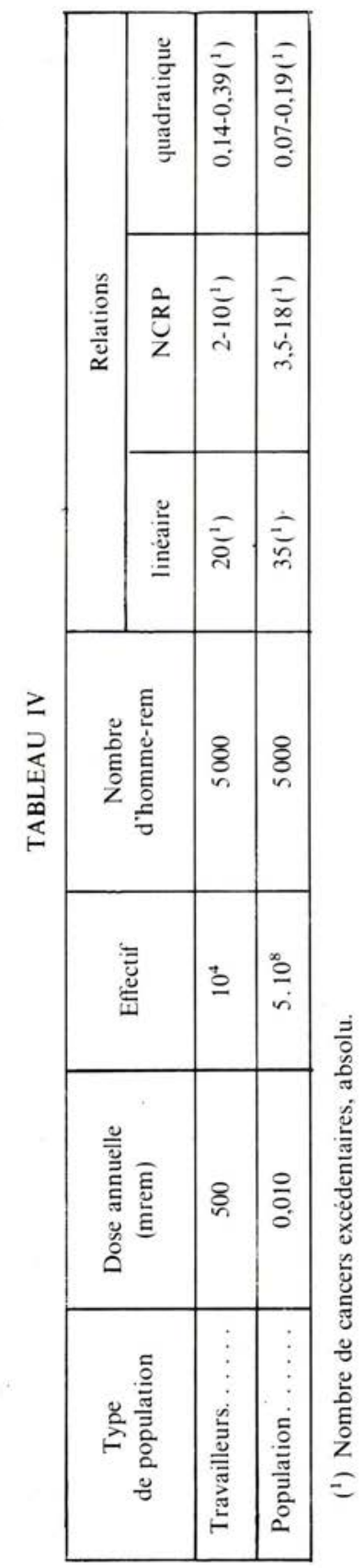


public « critique » supposé vivre près de la centrale et consommer eau, lait, poissons provenant des alentours de la centrale aboutit, dans les exemples étudiés, à une ou deux dizaines de microrems par an. Il s'agit, notons-le, d'un effectif peu important de population ainsi exposé, celui habitant dans un rayon de moins de $5 \mathrm{~km}$ de la centrale. Le tableau III donne deux de ces exemples tirés de la campagne 1980 .

Ces résultats très satisfaisants, puisque de l'ordre de quelques cent millièmes de la limite de dose autorisée au public, prouvent combien la protection de la population est correctement assurée.

Par contre, la dose collective des travailleurs, pour un effectif prévu vers 1990 de l'ordre de 10000 et une irradiation individuelle moyenne à 500 mrem par an, serait donc aux alentours de 5000 homme-rems. Sur la base des données ci-dessus de l'irradiation du public, pour aboutir à la même dose collective dans ce public, le calcul montre qu'il faudrait environ 300 millions de personnes, étant bien entendu que cette dose collective population n'aurait, de toute façon, pas la même signification que la première.

De plus, selon le choix du modèle de calcul, on peut inverser les conclusions concernant les dommages dans les deux cas. En effet, si l'application de la loi linéaire amène à une valeur de dommage supérieure dans le public à celle du groupe des travailleurs (35 au lieu de 20), par contre, le choix de la relation quadratique conduirait à un dommage deux fois plus faible et de toute façon non significatif, comme le montre le tableau IV. Il s'agit du nombre de cancers excédentaires attendus. Mais exprimés en risque relatif, les résultats changent totalement d'aspect et c'est un point très important :20 pour 10000 travailleurs, à comparer aux 2200 cancers attendus spontanément et 35 pour 500 millions de personnes, à rapporter aux 110 millions de cancers attendus (voir annexe).

Enfin, en raisonnant en équivalence de dommages calculés selon la relation quadratique, la population requise pour aboutir à l'équivalence avec le dommage pour 10000 travailleurs exposés à 500 mrem (soit $0,14-0,39$ ) serait, pour une irradiation de l'ordre de $10 \mu \mathrm{rem}$, exactement de 1 milliard ( $c f$. tableau II). Or, la population « critique » en France, même lorsque tous les sites nucléaires projetés seront en service, ne dépassera probablement pas quelques milliers de personnes : 10000 par exemple. On se trouve donc situé à une toute autre échelle. Pour aboutir à un nombre de cancers supérieur ou égal à 1 parmi cette population de 10000 personnes, le tableau II montre que, même en adoptant la relation dose-effet la plus majorante, il faudrait qu'elle soit exposée à plus de 10 mrem par an, du fait des rejets des centrales (à 10 mrem on aurait 0,7 cas). Le raisonnement à partir de la loi quadratique amènerait à fixer la limite d'irradiation bien plus haut encore. Par conséquent, l'effort de radioprotection doit porter en priorité sur les doses aux travailleurs.

\section{CONCLUSIONS}

La dose collective permet d'apprécier le coût dosimétrique de diverses opérations sur les sites nucléaires. Son utilité est toutefois limitée à l'étude à l'intérieur d'un même groupe exposé à des doses assez comparables entre elles. 
Additionner des fractions de millirem à des rems, lorsqu'on calcule une dose collective globale sur l'ensemble de la population française, nous paraît déjà peu logique, dans la mesure où il semble non fondé d'appliquer la relation linéaire aux niveaux de doses qui se confondent avec les fluctuations normales de l'irradiation naturelle. Ce calcul permet néanmoins d'estimer la limite supérieure de la dose collective.

A-t-on le droit de procéder de la même façon pour comparer les dommages ? Il nous semble, pour faire ces comparaisons, qu'il faille choisir les relations dose-effet les plus proches de la réalité pour les différents niveaux de dose : il s'agit, en effet, de dommages calculés à partir de modèles et, à ces niveaux, aucune vérification n'est possible. Étant donné les valeurs extrêmement faibles de l'irradiation de la population par les rejets des centrales, le bénéfice au plan sanitaire d'une réduction supplémentaire de cette irradiation serait infime, voire nul. Par contre, l'effort doit être soutenu vis-à-vis des travailleurs et, de toute façon, toute intercomparaison entre la dose collective de ceux-ci et celle de la population devrait être évitée, car elle est dépourvue de toute signification et risque d'aboutir à des conclusions totalement irréalistes et sans doute inexactes. Aussi, sans aller jusque-là, rappelons que Rossi exprime le vœu " qu'il y ait une réduction de l'importance, sinon une élimination totale des critères simplistes de la radioprotection, tels que " l'homme-rem ", « l'engagement de dose collectif » et autres concepts similaires, fondés sur des extrapolations linéaires jusqu'à des niveaux de dose inférieures au microgray (soit $0,1 \mathrm{mrad}$ ), et qui, selon le BEIR III, ne reposent sur aucun fondement scientifique ". Rappelons, enfin, que les calculs de dommages, à ces niveaux de dose, sont absolument invérifiables.

En conclusion, notre propos est simplement de rappeler que, si la notion de dose collective est évidemment très intéressante, il faut ne perdre de vue ni sa signification ni ses limites, ne pas en tirer des enseignements qu'elle ne peut fournir et ne pas faire de comparaisons évidemment discutables.

\section{ANNEXE}

\section{Risques absolus et relatifs}

\section{$1^{\circ}$ Risques population}

Pour une population de 500 millions de personnes, l'incidence moyenne de cancers est de $22 \%$ soit 110 millions. Les fluctuations statistiques à deux fois l'écart-type $\sigma$ sont au minimum :

$$
\mathrm{E}=2 \sigma=2 \sqrt{110.10^{6}}=20980 \text { cancers. }
$$

Le nombre de cancers surajoutés pour 5000 homme-rems est, suivant les relations dose-effet choisies :

- linéaire C.I.P.R. : 35 cancers soit $1,67 \cdot 10^{-3} \mathrm{E}$;

- linéaire N.C.R.P. : 3,5 à 18 cancers soit $1,67 \cdot 10^{-4} \mathrm{E}$ à $8,58 \cdot 10^{-4} \mathrm{E}$;

- quadratique Rossi : 0,07 à 0,19 cancer soit $3,34 \cdot 10^{-6} \mathrm{E}$ à $9,06 \cdot 10^{-6} \mathrm{E}$.

Aucune de ces variations ne sera observable. 


\section{$2^{\circ}$ Risques travailleurs}

Pour une population de travailleurs D.A.T.R. (*) de 10000 personnes, l'incidence moyenne attendue est de 2200 cancers. Les fluctuations statistiques sont alors :

$$
\mathrm{E}=2 \sigma=2 \sqrt{2200}=93,8 \text { cancers. }
$$

Le nombre de cancers surajoutés pour 5000 homme-rems est alors, suivant les relations dose-effet choisies :

- linéaire C.I.P.R. : 20 cancers soit $0,213 \mathrm{E}$;

- linéaire N.C.R.P. : 2 à 10 cancers soit $2,13 \cdot 10^{-2} \mathrm{E}$ à $0,107 \mathrm{E}$;

- quadratique Rossi : 0,14 à 0,39 cancer soit $1,49 \cdot 10^{-3} \mathrm{E}$ à $4,16 \cdot 10^{-3} \mathrm{E}$.

Le risque relatif n'est plus du même ordre de grandeur et on peut s'attendre, dans l'hypothèse de la C.I.P.R. mais non dans les autres, à observer des variations, même si elles ne sont pas significatives.

\section{BIBLIOGRAPHIE}

[1] Sindermann R. W. et Axtell C. E. Health Physics Society Midyear Topical Symposium, Los Angeles, 1969.

[2] International Commission on Radiological Protection (I.C.R.P.). Implications of Commission recommendations that doses be kept as low as readily achievable, ICRP Publication 22, Oxford, Pergamon Press, 1973.

[3] International Commission on Radiological Protection (I.C.R.P.), Recommendations, ICRP Publication 26, Oxford, Pergamon Press, 1977.

[4] United Nations Scientific committee on the effects of atomic radiation (U.N.S.C.E.A.R.). Sources and effects of ionizing radiations, New York, United Nations, 1977.

[5] National council on radiation protection and measurements (N.C.R.P.). Influence of dose and on dose-response relationship its distribution in time for low-LET-radiations, N.C.R.P. report 64, Washington, N.C.R.P., 1980.

[6] Advisory committee on the biological effects of ionizing radiations. The effects on populations of exposure to low levels of ionizing radiation, BEIR III, Washington, National Academy of Sciences, 1980.

[7] Advisory committee on the biological effects of ionizing radiations. The effects on populations of exposure to low levels of ionizing radiation, BEIR I, Washington, National Academy of Sciences, 1972.

(*) D.A.T.R. : directement affectés à des travaux sous rayonnement. 